\title{
The Management of Bleeding from Anorectal Varices
}

\author{
Marcus Robertson ${ }^{1,2}$ • Alexandra Ines Thompson ${ }^{1} \cdot$ Peter Clive Hayes ${ }^{1}$
}

Published online: 7 November 2017

(C) The Author(s) 2017. This article is an open access publication

\begin{abstract}
Purpose of Review The purpose of this review is to summarize available strategies for the diagnosis and management of bleeding anorectal varices.

Recent Findings Interventional radiological procedures, including TIPS, BRTO and/or embolization, have been established as efficacious treatments, particularly in the setting of treatment failure.

Summary Anorectal varices are prevalent in patients with portal hypertension. Acute bleeding is uncommon, but can be massive and life-threatening. Anorectal varices should be considered as a differential diagnosis in any patient with cirrhosis or portal hypertension who presents with lower gastrointestinal bleeding. No evidence-based guidelines exist to guide the management of bleeding anorectal varices, which typically requires a multidisciplinary team of endoscopists, hepatologists, surgeons and interventional radiologists. Administration of prophylactic antibiotics and vasoactive therapy is recommended based on efficacy in bleeding oesophageal varices. Urgent endoscopy should be performed in all patients. Endoscopic sclerotherapy has the greatest body of evidence and should be considered the first-line therapy; efficacy and safety may be increased if this is performed with endoscopic ultrasound. Endoscopic banding or obturation are
\end{abstract}

Marcus Robertson and Alexandra Ines Thompson joint first-author

This article is part of the Topical Collection on Portal Hypertension

Peter Clive Hayes

P.Hayes@ed.ac.uk

1 Department of Hepatology, Royal Infirmary of Edinburgh, Edinburgh, UK

2 Department of Gastroenterology, Monash Health, Melbourne, Australia alternative first-line treatments; all methods offer a technically simple and efficacious method of achieving haemostasis, and local expertise will determine which procedure is employed.

Keywords Anorectal varices · Endoscopy $\cdot$ Cirrhosis $\cdot$ Portal hypertension $\cdot$ Band ligation $\cdot$ Sclerotherapy

\section{Introduction}

Variceal bleeding is a common and life-threatening manifestation of portal hypertension and remains an important cause of death in patients with cirrhosis [1]. Esophago-gastric varices are by far the most common cause of acute variceal bleeding (AVB), the management of which is well-established and evidence-based. Ectopic varices are defined as dilated portosystemic collateral veins occurring anywhere in the gastrointestinal tract other than the esophago-gastric region and include duodenal, jejunal, ileal, stomal, colonic and anorectal varices [2]. Ectopic varices constitute $2-5 \%$ of all variceal bleeds but are the cause of bleeding in 20-30\% of patients with extrahepatic portal hypertension [3-5]. Although rare, acute ectopic variceal bleeding can be massive and life-threatening. The diagnosis and management of bleeding ectopic varices remains challenging, and their anatomical diversity makes development of standardized guidelines extremely difficult.

Anorectal varices represent portal-systemic collaterals manifesting as discrete, dilated submucosal veins, extending proximal to the dentate line and into the rectum [6]. Bleeding rectal varices typically present as hematochezia; an acute bleed from anorectal varices is rare but may result in lifethreatening haemorrhage. This review aims to summarize available strategies for the diagnosis and management of bleeding anorectal varices. 


\section{Pathophysiology, Aetiology and Prevalence of Anorectal Varices}

Portal hypertension is a well-recognized and common manifestation of chronic liver disease. In portal hypertension, a combination of increased splanchnic blood flow and intrahepatic resistance to portal blood flow leads to a pathological increase in portal pressures, which results in the development of portosystemic collaterals, the most clinically significant of which are gastroesophageal varices [7]. The hepatic venous pressure gradient (HVPG) is a useful clinical measure of portal pressures and is defined as the gradient between the wedged hepatic venous pressure and the free hepatic venous pressure [8-10]. The HVPG can be used to risk stratify patients with portal hypertension, although in practice it is not frequently performed due to the invasiveness of the procedure. The normal HVPG ranges between 1 and $5 \mathrm{mmHg}$ and a portal pressure gradient of $\geq 12 \mathrm{mmHg}$ is well established as the baseline elevated pressure above which variceal development and bleeding may occur.

Anorectal varices represent porto-systemic collateral vessels that constitute a pathway for portal venous blood flow between the superior rectal veins of the inferior mesenteric system and the middle inferior rectal veins of the iliac system $[2,11,12]$. They manifest as dilated and engorged submucosal veins in the rectum [6]. Anorectal varices most commonly result from portal hypertension secondary to cirrhosis [13]. A variety of conditions that result in non-cirrhotic portal hypertension are also associated with the development of anorectal varices [14], including mesenteric [15-17] or splenic vein obstruction [18] from carcinoid syndrome or pancreatitis respectively, along with cavernous malformation of the portal vein. In addition, systemic conditions such as congestive heart failure or congenital vascular anomalies have been postulated as potential causes of anorectal varices [6].

The incidence of anorectal varices varies widely between studies, ranging from 38 to $95 \%$ [19, 20]. In patients with established liver cirrhosis, prevalence ranges from 38 to $92 \%[19,21]$. Multiple studies have suggested a higher prevalence of anorectal varices in patients with non-cirrhotic portal hypertension or extra-hepatic portal vein obstruction (63$95 \%$ ) and varices were significantly larger in this cohort [19, 20]; no identifiable causes have been found to explain these differences [6]. In addition, Hosking et al. demonstrated in a prospective trial that the incidence of anorectal varices may be related to duration of portal hypertension, with a $19 \%$ incidence in patients with early cirrhosis, increasing to $59 \%$ in patients with a long duration of portal hypertension [22].

A large study by Watabane et al. found that anorectal varices were the most common site of ectopic varices, constituting $45 \%$ of the cohort. Furthermore, patients with rectal varices were frequently noted to have a history of esophageal varices $(94.8 \%)$ and the majority $(87 \%)$ had received endoscopic treatment of esophageal varices [23]. There is conflicting evidence regarding the occurrence of rectal varices following treatment of esophageal varices. Some studies have suggested that injection sclerotherapy or band ligation of esophageal varices may influence the natural history of ectopic varices. In the case of anorectal varices, this is postulated to be due to development of collateral vessels in the inferior mesenteric venous system following obliteration of supplying vessels (such as the left gastric, posterior gastric or short gastric veins) which ultimately leads to the formation of rectal varices $[11,23,24]$. The correlation between treatment of esophageal or gastric varices and subsequent formation of anorectal varices has not been conclusively demonstrated.

Despite the high prevalence of anorectal varices, massive haemorrhage remains an uncommon event and studies report a frequency ranging from 0.5 to $3.6 \%$ [25]. The prevalence of haemorrhage from anorectal varices is significantly increased in larger varices and in patients with high-risk stigmata such as a positive "red colour" sign [26]. There is no evidence to suggest that the incidence of bleeding is increased with the presence of oesophageal variceal bleeding, a history of previous treatment of oesophageal varices or the aetiology of portal hypertension $[6,26]$.

\section{Clinical Presentation, Diagnosis and Evaluation of Anorectal Varices}

Bleeding anorectal varices most commonly presents as hematochezia (bleeding per rectum), which is typically acute or chronic recurrent. The diagnosis of anorectal varices is usually made at endoscopy (Fig. 1); however, endoscopic ultrasound (EUS) and imaging have also been employed.

\section{Endoscopy}

Diagnosis of anorectal variceal bleeding is primarily achieved at endoscopy (either flexible sigmoidoscopy or colonoscopy) [25], which remains the gold-standard investigation. Anorectal varices typically appear as blue-tinted, serpentine, sub-mucosal varicose veins located near the anus, which always cross the dentate line to extend cranially and can also extend into the rectum $[6,11,22]$. Diagnosis can be difficult in the setting of massive gastrointestinal bleeding and a highindex of suspicion is vital whenever a patient with known portal hypertension presents with rectal bleeding. Patients with anorectal varices may also have other lower gastrointestinal sources of bleeding, and thus, a colonoscopy may be required to accurately locate the bleeding point.

A critical task at endoscopy is to differentiate between anorectal varices and haemorrhoids, which can be difficult in the setting of active bleeding. Unlike anorectal varices, the prevalence of haemorrhoids is not increased in patients with 

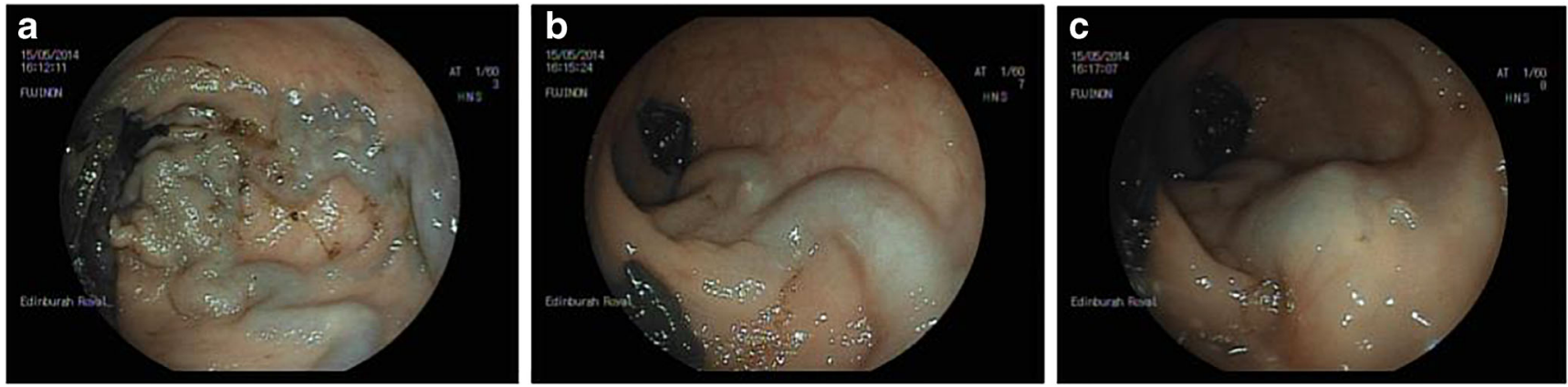

Fig. 1 a A leash of varices in the rectum. b A rectal varix. c Varix in b post injection with thrombin. Note puncture site

portal hypertension [19]. Maslekar et al. eloquently summarized endoscopic criteria to differentiate anorectal varices from haemorrhoids (Table 1); a notable difference is that anorectal varices, unlike haemorrhoids, are compressible and refill immediately on release [6].

\section{Endoscopic Ultrasound (EUS)}

Conventional EUS has been demonstrated to be a useful modality for the hemodynamic diagnosis and evaluation of anorectal varices in both children [27] and adults [28, 29]. Indeed, EUS has been shown to be superior to endoscopy in detecting the presence and number of rectal varices [25]. Dhiman et al. found EUS to have superior diagnostic accuracy to endoscopy, finding rectal varices in $75 \%$ patients with portal hypertension compared to $43 \%$ via endoscopy [29]. EUS can also detect deep rectal varices in a large proportion of patients who do not have identified varices on routine endoscopy [30], although the significance of these remains uncertain.

Endoscopic colour doppler ultrasonography (ECDUS) has also been shown to be useful in the evaluation of anorectal varices. ECDUS can detect anorectal varices through colour flow images, and it facilitates more detailed observation of the hemodynamics of rectal varices than conventional EUS [31]. In addition, ECDUS may be helpful in risk stratifying anorectal varices through measurement of the velocity of blood flow within a varix; Sato et al. demonstrated a significantly higher mean velocity of blood flow in the rectal varices of patients experiencing acute bleeding compared to patients who did not experience bleeding [32]. Endoscopic treatments

Table 1 Features at endoscopy that help to differentiate between anorectal varices and haemorrhoids [6]

\begin{tabular}{lll}
\hline Feature & Anorectal varices & Haemorrhoids \\
\hline Site & Rectum + anal canal & Anal canal \\
Colour & Bluish-grey & Purplish \\
Prolapse & No & Possible \\
Compressibility & Yes & No \\
\hline
\end{tabular}

such as injection sclerotherapy can also be performed with ECDUS, which can assess efficacy by a reduction in the velocity of blood flow in rectal varices following injection [33].

\section{Imaging}

Historically, barium enema had been used as a diagnostic tool for anorectal varices, but this procedure is obsolete in the era of endoscopy. MRI-venography is a non-invasive method of demonstrating anorectal varices, along with varices at other sites, and it has been successfully used to assess patients preand post-transjugular intrahepatic portosystemic shunt (TIPS) insertion [34].

\section{Management of Bleeding Anorectal Varices}

In contrast to the management of esophageal varices, where well-refined and evidence-based standards of care have been developed, the optimal treatment for anorectal varices remains to be determined. No randomized control trials or prospective studies exist to guide management of anorectal varices, and publications are limited to case reports or small case series. In addition, heterogeneity between studies and small patient numbers makes comparisons of potential treatments extremely difficult. A variety of treatment modalities have successfully been employed to treat bleeding anorectal varices, including:

- Endoscopic therapies (endoscopic injection sclerotherapy (EIS), band ligation (EBL) or obturation (EVO))

- Interventional radiological procedures (TIPS, balloonoccluded retrograde trans-venous obliteration (BRTO), embolization)

- Surgical procedures (including simple suture ligation or stapled anopexy, mesenteric vein occlusion or porto-caval shunt surgery)

In all cases of ectopic variceal haemorrhage, a multidisciplinary team of endoscopists, hepatologists, surgeons and interventional radiologists may be required. The most appropriate 
therapeutic modality may depend on the clinical condition of the patient, the cause of portal hypertension, and locally available expertise or facilities. Management may also include transfer to a tertiary referral centre for specialized therapy [7]. A proposed treatment algorithm is presented in Fig. 2.

\section{Assessment and Medical Management}

Primary therapeutic goals in any patient presenting with variceal bleeding include urgent hemodynamic resuscitation, prevention and treatment of complications, and early endoscopic intervention to localize bleeding and induce haemostasis. Fluid resuscitation should be initiated as soon as possible aiming to maintain a systolic blood pressure around 90 to $100 \mathrm{mmHg}$. Prolonged periods of hypotension should be avoided to prevent complications such as infection and renal failure, which are associated with increased morbidity and mortality and a higher risk of rebleeding $[35,36]$. As with other forms of gastrointestinal bleeding, transfusion of blood should be performed
Fig. 2 Proposed treatment algorithm for bleeding anorectal varices

Haemodynamic resuscitation
Large-bore intravenous or central line access
Fluid resuscitation aiming to maintain SBP $90 \mathrm{mmHg}$
Conservative blood transfusion policy (Target $\mathrm{Hb} 7-8 \mathrm{~g} / \mathrm{dL}$ )
Pneumatic tamponade of varices with a Foley's catheter, Sengstaken-Blakemore
tube or rectal balloon can be used as a temporising measure prior to endoscopy in
patients with active bleeding

\section{Prophylactic antibiotic therapy}

Antibiotics improve survival and prevent infection in bleeding oesophageal varices; efficacy in bleeding anorectal varices is inferred

All patients receive $3-5$ days of a broad-spectrum antibiotic

\section{Vasoactive pharmacological therapy}

Vasoactive medications commonly commenced prior to endoscopy in patients with suspected oesophago-gastric varices and continued for 3-5 days. Agents include Terlipressin or Octreotide

Empiric use in bleeding anorectal varices is inferred from efficacy in oesophageal varices

\section{Endoscopic therapy}

Urgent flexible sigmoidoscopy or colonoscopy is recommended in all patients

Endoscopic injection sclerotherapy (EIS) has the greatest body of evidence and should be considered a first-line treatment option; efficacy and safety may be improved if EIS is performed with fluoroscopy or endoscopic ultrasound (EUS)

Endoscopic variceal ligation (EVL, "banding") and variceal obturation (EVO) with either glue or thrombin are alternative first-line treatments

The choice of endoscopic treatment employed will depend on operator familiarity and expertise

\section{Second-line and salvage therapies}

If refractory ongoing bleeding despite endoscopic and pharmacologic therapy, second-line and salvage treatment options include:

- Angiographic embolization: High rebleeding rate noted with monotherapy

- Transjugular intrahepatic portosystemic shunt (TIPS): Often performed in conjunction with embolization; contraindicated if Child-Pugh $>13$ or significant hepatic encephalopathy

- Balloon-occluded retrograde trans-venous obliteration (BRTO): Minimally invasive but highly-specialised procedure with limited evidence in bleeding anorectal varices

- Surgical procedures: Rarely performed 
cautiously using a restrictive strategy aiming to maintain the haemoglobin level between 7 and $8 \mathrm{~g} / \mathrm{dl}$; this is associated with significantly improved outcomes in patients with acute upper gastrointestinal bleeding [37]. Patients with rapid ongoing bleeding and those with underlying ischemic heart disease may benefit from a more liberal transfusion policy. Correction of coagulopathy is commonly performed but remains contentious and this should not delay endoscopy.

Both vasoactive therapy (such as terlipressin or octreotide) and empiric antibiotic administration are commonly used in anorectal variceal bleeding, although there are no specific data to support these interventions in ectopic variceal bleeding. Vasoactive drugs aim to decrease splanchnic blood flow and portal pressure and are efficacious in the management of bleeding oesophageal varices [38]. In addition, empiric administration of antibiotics is considered standard of care in all cirrhotic patients presenting with GI bleeding [39-41] and significantly reduces the incidence of infection, resulting in decreased risk of rebleeding [42], all-cause mortality [43] and hospital length of stay. Both vasoactive therapy and antibiotics have an excellent safety profile, and their efficacy in anorectal variceal bleeding is inferred from their successful use in the management of esophageal varices.

\section{Pneumatic Tamponade of Varices}

Although not published, our centre has successfully employed pneumatic tamponade as a temporizing measure in the management of anorectal varices. Instruments such as a Foley's catheter, Sengstaken-Blakemore tube or a rectal tube with balloon can be used to pneumatically compress acutely bleeding anorectal varices prior to endoscopy; this can also facilitate transfer to a specialist centre. The balloon is inflated in the rectum and gentle traction employed to compress the anorectal varices; the instrument can be fastened to the patient's thigh to facilitate constant traction.

\section{Endoscopic Management of Anorectal Varices}

Endoscopic interventions are generally considered first-line in the management of acutely bleeding anorectal varices and are also used for secondary prophylaxis.

\section{Endoscopic Injection Sclerotherapy (EIS)}

The use of EIS to successfully treat bleeding anorectal varices was first described by Wang et al. in 1985 [44], and other case reports of successful EIS for treatment of bleeding anorectal varices have subsequently been published [45-47]. Whilst EIS is considered a first-line treatment for bleeding anorectal varices, there is no current recommendation in relation to the concentration and volume of sclerosant to be injected. Sato et al. performed EIS using 5\% ethanolamine oleate with iopamidol, in 25 patients (14 patients with a history of rectal bleeding and 11 patients at high risk of variceal bleeding based on endoscopic findings) [48]. EIS was performed weekly, a mean of 2.7 times, with a mean total sclerosant volume of $5.2 \mathrm{~mL}$ injected per procedure. Whilst complications of erosions and ulceration have been associated with EIS of anorectal varices [44, 45], none were observed in this series. These patients were compared to nine patients with anorectal varices treated with EBL. Overall, the recurrence rate of anorectal varices over a 1-year follow-up period was lower in the EIS group (33.3\% vs. $55.6 \%$ ), although this difference was not significant. Of note, however, recurrence of anorectal variceal bleeding was significantly higher in the EBL group [48]. This remains the only study comparing EIS and EBL in the management of anorectal varices and suggests that EIS may be favourable to EBL in terms of long-term effectiveness and complications; it is important to remember that patient numbers were very small and results should be interpreted with caution. Larger studies are required before evidencebased treatment recommendations can be developed. In addition, as EIS has long been superseded by EBL in the management of esophageal varices, operator familiarity with EIS may be low which can potentially increase the risk of adverse events.

Haemodynamic evaluation of anorectal varices prior to EIS has been recommended to minimize the risk of serious complications such as vascular embolism. Sato et al. recommended injecting the sclerosant slowly under fluoroscopic guidance with careful monitoring to ensure the sclerosing agent does not flow into the systemic circulation; the risk of this is elevated when blood flow in a rectal varix is high [48].

\section{Endoscopic Band Ligation (EBL)}

EBL remains the gold-standard treatment for bleeding esophageal varices and was first used for bleeding anorectal varices by Kojima et al. in 1996 [49]. Uno et al. subsequently described successful treatment of bleeding anorectal varices by EBL in a child with extrahepatic portal hypertension following failure of EIS; bleeding did not recur over 13 months of follow-up [50]. The long-term efficacy of EBL was demonstrated by Firoozi et al., who followed a patient for 46 months following successful use of EBL to treat and obliterate bleeding anorectal varices, observing no further bleeding [51]. EBL has been described as a safe and effective therapy for anorectal varices [52].

EBL is an attractive therapeutic option, particularly as it is a familiar technique widely used in management of oesophageal varices, and it appears to be an effective first-line treatment for anorectal variceal bleeding. Similar to gastric varices, EBL may not be an optimal therapy for large anorectal varices 
and if the diameter of the varix exceeds the diameter of the endoscope, EBL should probably be avoided [4]. If the entire varix cannot be banded, there may be a high risk of developing a wide defect in the varix, especially after sloughing, increasing the risk of post-banding bleeding [6].

In addition, some investigators have reported that rectal varices can easily recur following EBL $[25,53]$. CoelhoPrabhu et al. presented a retrospective case series of 10 consecutive patients who underwent EBL for bleeding anorectal varices [54]. No procedural complications were experienced; however, four patients experienced a rebleeding event; in three patients, this was successfully managed with repeat EBL and one patient died from uncontrolled haemorrhage. In this study, the average number of bands used was 4 (range of 1-11) and bands were always placed as distally as possible, aiming close to the anal verge. Use of more bands at the index endoscopy was not associated with a reduction in the number of subsequent procedures required for variceal management. The authors concluded that EBL was successful in this small cohort, although the majority required more than one treatment [54]. Currently, the literature in relation to EBL for anorectal varices is limited to case reports or small case series, and larger trials are necessary to accurately characterize rebleeding rates and complications such as bleeding from procedure-related ulcers.

\section{Endoscopic Variceal Obturation (EVO)}

Injection of cyanoacrylate glue is an accepted therapy for bleeding gastric varices, and the technique has successfully been used to achieve haemostasis in bleeding anorectal varices [55-57]. Obturation is the term used for varices treated by cyanoacrylate (glue) injection because the varix remains visible as a hardened structure after successful treatment [58]. Tissue adhesive such as $n$-butyl-2-cyanoacrylate are injected into the varix lumen and undergo rapid polymerization upon contact with blood (changing from a liquid to a hard acrylic plastic), ultimately resulting in vascular obstruction [59].

Experience of EVO in the management of anorectal varices is again limited to case reports. Weilert et al. reported successful management of bleeding rectal varices with EUS-guided cyanoacrylate injection in conjunction with intraluminal placement of embolization coils [56]. Multiple other case reports have also described EUS-guided EVO as a promising and effective modality in the management of bleeding rectal varices [60-62]. Conventional EVO with a traditional endoscope may not be feasible in all cases due to poor visualization and inadvertent missing of the variceal source of bleed; although the evidence base is small, EUS-guided EVO may be more successful in the setting of bleeding anorectal varices. Sharma et al. have also reported EUS-guided histoacryl glue injection to treat significant bleeding from submucosal anorectal varices that were not evident by endoscopy alone [57].
It is known from experience with gastric varices that glue injection can be associated with serious adverse events such as systemic embolization and sepsis; the risk of this is thought to be related to the volume of glue injected [63]. Post-procedure thromboembolic phenomena are well-documented following glue injection, including pulmonary embolism, cerebral stroke, portal vein embolization and infarction in multiple organs $[11,63]$. The use of coils to provide a scaffold to retain glue within the varix is postulated to minimize the risk of embolization and may facilitate variceal obliteration with a smaller volume of glue injection $[11,56]$.

\section{Thrombin Injection}

The haemostatic agent thrombin converts fibrinogen to fibrin clot and enhances platelet aggregation. It has been used extensively for management of gastric varices, although there are still no randomized controlled trials evaluating the efficacy of thrombin versus EVO. Human thrombin is now used rather than bovine thrombin, due to risk of prion transmission [7]. In a large case series evaluating the efficacy and safety of thrombin injection for bleeding gastric and ectopic varices, McAvoy et al. demonstrated a $100 \%$ rate of initial haemostasis and a $10 \%$ rebleeding rate. Only one patient with bleeding rectal varices was treated in this series; an absolute volume of $18.3 \mathrm{ml}$ thrombin was used over 3 endoscopy sessions, and haemostasis was achieved with no rebleeding encountered [64]. Our centre uses thrombin injection as a first-line therapy for bleeding rectal varices and has found it to be highly efficacious; human thrombin at a concentration of $250 \mathrm{IU}$ per $\mathrm{mL}$ is used with up to $10 \mathrm{~mL}$ injected per endoscopy session.

\section{Interventional Radiological Procedures}

\section{Embolization Therapy}

Embolization therapy is a radiological technique that can be performed to occlude the feeding vein to anorectal varices [65]. A variety of different embolization materials are available, including coils, gelfoam, thrombin, collagen, autologous blood clot and ethanol. Because angiographic embolization does not lower portal pressures, high rebleeding rates have been observed with monotherapy and combination therapy with TIPS is often recommended [7]. A case report by Anh et al. demonstrated successful variceal embolization of anorectal varices following rebleeding after TIPS [66].

\section{TIPS}

The radiologically placed transjugular intrahepatic portosystemic shunt (TIPS) is effective in achieving 
haemostasis in about $95 \%$ of patients with refractory variceal bleeding [8]. Multiple publications have reported the successful use of TIPS in controlling bleeding ectopic varices, which has typically been employed as a salvage therapy. The use of TIPS in a patient with refractory anorectal variceal bleeding was first documented by Katz et al. in 1993. Marked decompression of the varices was noted $24 \mathrm{~h}$ following TIPS placement and no recurrent bleeding recorded after 6 months of follow-up [34].

There are now a number of case reports and small case series describing successful management of anorectal varices with TIPS [67-70]. Larger case series have also demonstrated TIPS to be a highly effective modality for controlling bleeding [71-75], although there are multiple reports of ectopic variceal rebleeding despite a reduction in the HVPG to $<12 \mathrm{mmHg}$. Thus, a combination of TIPS and other treatment modalities such as embolization or endoscopic therapy is recommended wherever possible to effectively control haemorrhage from anorectal varices $[71,75]$.

TIPS has several attractive advantages: it is a highly efficacious but minimally invasive procedure that can be performed in a single session without the need for general anaesthesia. In addition, TIPS placement is not a contraindication to liver transplantation. Thus, TIPS may be used in the setting of acute bleeding both as a bridge to transplantation or as a definitive therapy in patients unfit for surgery [6]. Following successful TIPS placement, the long-term survival of patients is largely determined by their underlying liver function and, thus, careful patient selection is critical; the procedure is largely contraindicated in patients with a Child-Pugh score $>13$. The potential benefits of TIPS must be weighed against risks, which include an increased risk of encephalopathy, recurrent bleeding, procedure-related morbidity and a 30-day mortality of between 3 and $15 \%$ [6, 34, 69].

\section{BRTO}

BRTO is an advanced radiological procedure first described by Kanagawa et al. [76] in 1996 for management of gastric varices. Gastric varices often have unique vascular anatomy, with spontaneous spleno-renal or gastro-renal shunts (GRS) that divert blood flow into the systemic circulation [77]. This provides a pathway for interventional radiologists to access and facilitate transvenous obliteration of the portosystemic shunts. In BRTO, an occlusion balloon is used to isolate gastric varices and collateral veins, followed by endovascular injection of a sclerosing agent and/or microcoils directly into the gastro-variceal system, resulting in variceal obliteration [78]. A small randomized study by Choi et al. compared BRTO with TIPS for treatment of active gastric variceal bleeding and found no differences with regard to rates of hemostasis, rebleeding, or encephalopathy [79]. BRTO has some potential advantages over TIPS: it is less invasive, can be performed in patients with advanced liver disease and is suitable in patients with hepatic encephalopathy [80]. Adverse complications of BRTO include haemoglobinuria, abdominal pain, pyrexia, and pleural effusion [58]. In addition, hepatic portal blood flow and portal pressures have been shown to increase after BRTO. This may improve liver function $(50 \%$ of patients had an improvement in Child-Pugh score in one study), but can worsen the size of varices at other sites (potentially increasing the risk of bleeding) and may exacerbate ascites [81-84]. Hepatic encephalopathy may also improve following BRTO, but whether this beneficial effect is sustained long-term remains unknown [83].

Anan et al. reported the use of BRTO to successfully treat bleeding colonic varices in a patient with hepatic encephalopathy. BRTO resulted in resolution of encephalopathy but was noted to worsen pre-existing oesophageal varices. More recently, BRTO was shown to be successful in conjunction with surgical suturing, in controlling bleeding rectal varices with a large $(1.26 \mathrm{~cm})$ feeding vessel, although the patient died 6 months post procedure from liver failure [85].

\section{Surgical Management}

Surgical procedures for bleeding anorectal varices are generally only considered when endoscopic modalities or interventional embolization techniques have failed to induce haemostasis and are rarely performed. Surgical methods include simple suture ligation and stapled anopexy, mesenteric vein occlusion and porto-caval shunt surgery.

\section{Suture Ligation and Stapled Anopexy}

Direct suture ligation is a technically challenging procedure with questionable efficacy [6]. Stapled anopexy has been suggested as a simple technique that could be employed in treating bleeding anorectal varices if EBL or EIS fails. It is thought that this procedure may be effective due to disruption of portosystemic connections in the anorectum [6]. Stapled anopexy was described by Biswas et al., who placed a purse-string suture $4 \mathrm{~cm}$ above the dentate line to successfully halt massive bleeding from anorectal varices [86]. Botterill et al. used a circumferential stapling device to induce haemostasis in a patient with bleeding anorectal varices who had failed both EBL and EIS [87]. A subsequent case series of nine patients by Kaul and Skaife. also demonstrated that stapling can be an efficacious method of treating bleeding anorectal varices when carried out by an experienced colorectal surgeon, with no rebleeding encountered [88].

Mesenteric Vein Ligation In patients with intractable anorectal variceal bleeding, mesenteric vein ligation has been described as an alternative surgical option to induce 
haemostasis. Instant variceal decompression and cessation of bleeding was demonstrated in a case report by Yeh and McGuire [89], although this procedure is rarely performed.

Portocaval Shunt Surgery If endoscopic and/or interventional radiologic procedures fail to control bleeding or are not feasible, surgery is a potential option if the expertise is available. Portocaval shunt surgery has been shown to be very effective in controlling life-threatening haemorrhage, but careful patient selection is imperative as mortality is extremely high (up to $80 \%$ ), primary due to liver failure. Surgery is preferred in patients with Child-Pugh class A cirrhosis and in those with extra-hepatic portal vein occlusion [90]. There is generally little role for surgical procedures in the current management of bleeding anorectal varices.

\section{Conclusion}

Anorectal varices are prevalent in patients with portal hypertension; acute bleeding is uncommon but can be massive and life-threatening when it occurs. Anorectal varices should be considered as a differential diagnosis in any patient with cirrhosis or portal hypertension who presents with lower gastrointestinal bleeding. The management of bleeding anorectal varices requires a multidisciplinary team of endoscopists, hepatologists, surgeons and interventional radiologists; however, due to a paucity of studies, no evidence-based guidelines exist to guide management. Urgent endoscopy should be performed in all patients. Endoscopic injection sclerotherapy has the greatest body of evidence as a first-line treatment; endoscopic banding or obturation are alternative first-line treatments. All three techniques offer an easy and efficacious method of obtaining early haemostasis and the decision of which procedure will depend on local expertise. Administration of prophylactic antibiotics and vasoactive therapy is also recommended based on efficacy in bleeding oesophageal varices. In the setting of treatment failure, a variety of second-line treatment options can be employed, including TIPS, BRTO or surgery.

Open Access This article is distributed under the terms of the Creative Commons Attribution 4.0 International License (http:// creativecommons.org/licenses/by/4.0/), which permits unrestricted use, distribution, and reproduction in any medium, provided you give appropriate credit to the original author(s) and the source, provide a link to the Creative Commons license, and indicate if changes were made.

\section{References}

1. Graham DY, Smith JL. The course of patients after variceal hemorrhage. Gastroenterology. 1981;80(4):800-9.
2. Sato T, Akaike J, Toyota J, Karino Y, Ohmura T. Clinicopathological features and treatment of ectopic varices with portal hypertension. Int J Hepatol. 2011;2011:1-9.

3. Lebrec D, Benhamou JP. Ectopic varices in portal hypertension. Clin Gastroenterol. 1985;14(1):105-21.

4. Norton ID, Andrews JC, Kamath PS. Management of ectopic varices. Hepatology. 1998;28(4):1154-8.

5. Henry Z, Uppal D, Saad W, Caldwell S. Gastric and ectopic varices. Clin Liver Dis. 2014;18(2):371-88.

6. Maslekar S, Toh E-W, Adair R, Bate JP, Botterill I. Systematic review of anorectal varices. Color Dis. 2013;15(12):e702-10.

7. Robertson M, Hayes P. Management of acute variceal bleeding. In: Plevris JN, Hayes PC, Kamath PS, Wong Kee Song LM, editors. Endoscopy in liver disease. Oxford: John Wiley \& Sons; 2018.

8. Ferguson JW, Tripathi D, Hayes PC. Review article: the management of acute variceal bleeding. Aliment Pharmacol Ther. 2003;18(3):253-62.

9. Perelló A, Escorsell A, Bru C, Gilabert R, Moitinho E, GarcíaPagán JC, et al. Wedged hepatic venous pressure adequately reflects portal pressure in hepatitis $\mathrm{C}$ virus-related cirrhosis. Hepatology. 1999;30(6):1393-7.

10. Boyer TD, Triger DR, Horisawa M, Redeker AG, Reynolds TB. Direct transhepatic measurement of portal vein pressure using a thin needle. Comparison with wedged hepatic vein pressure. Gastroenterology. 1977;72(4 Pt 1):584-9.

11. Al Khalloufi K, Laiyemo AO. Management of rectal varices in portal hypertension. World J Hepatol. 2015;7(30):2992-8.

12. Sato T. Treatment of ectopic varices with portal hypertension. World J Hepatol. 2015;7(12):1601-5.

13. Gudjonsson H, Zeiler D, Gamelli RL, Kaye MD. Colonic varices. Report of an unusual case diagnosed by radionuclide scanning, with review of the literature. Gastroenterology. 1986;91(6):1543-7.

14. Orozco H, Takahashi T, Mercado MA, Prado-Orozco E, Ferral H, Hernandez-Ortiz J, et al. Colorectal variceal bleeding in patients with extrahepatic portal vein thrombosis and idiopathic portal hypertension. J Clin Gastroenterol. 1992;14(2):139-43.

15. Manzi D, Samanta AK. Adhesion-related colonic varices. J Clin Gastroenterol. 1985;7(1):71-5.

16. Granqvist S. Colonic varices caused by carcinoid tumor. Gastrointest Radiol. 1984;9(3):269-71.

17. Miao YM, Catnach SM, Barrison IG, O'Reilly A, Divers AR. Colonic variceal bleeding in a patient with mesenteric venous obstruction due to an ileal carcinoid tumour. Eur J Gastroenterol Hepatol. 1996;8(11):1133-5.

18. Vella-Camilleri FC, Friedrich R, Vento AO. Diffuse colonic varices: an uncommon cause of intestinal bleeding. Am J Gastroenterol. 1986;81(6):492-4.

19. Misra SP, Dwivedi M, Misra V. Prevalence and factors influencing hemorrhoids, anorectal varices, and colopathy in patients with portal hypertension. Endoscopy. 1996;28(4):340-5.

20. Chawla Y, Dilawari JB. Anorectal varices - their frequency in cirrhotic and non-cirrhotic portal hypertension. Gut. 1991 Mar;32(3): 309-11.

21. Goenka MK, Kochhar R, Nagi B, Mehta SK. Rectosigmoid varices and other mucosal changes in patients with portal hypertension. Am J Gastroenterol. 1991;86(9):1185-9.

22. Hosking SW, Smart HL, Johnson AG, Triger DR. Anorectal varices, haemorrhoids, and portal hypertension. Lancet. 1989;1(8634): $349-52$.

23. Watanabe N, Toyonaga A, Kojima S, Takashimizu S, Oho K, Kokubu S, et al. Current status of ectopic varices in Japan: results of a survey by the Japan Society for Portal Hypertension. Hepatol Res. 2010;40(8):763-76.

24. Frossard JL, Seirafi M, Spahr L. Ectopic varices and collaterals development after band ligation treatment in a patient with portal hypertension. Case Rep Gastroenterol. 2008;2(3):380-3. 
25. Sato T, Yamazaki K, Akaike J. Diagnosis and Endoscopic Treatments of Rectal Varices. In: Joaquim J, da Rocha R, editors. Endoscopic procedures in colon and rectum. InTech;2011. Available at: http://cdn.intechopen.com/pdfs/22627/InTechDiagnosis and endoscopic treatments of rectal varices.pdf

26. Shudo R, Yazaki Y, Sakurai S, Uenishi $\bar{H}$, Yamada H, Sugawara K. Clinical study comparing bleeding and nonbleeding rectal varices. Endoscopy. 2002;34(3):189-94.

27. Yachha SK, Dhiman RK, Gupta R, Ghoshal UC. Endosonographic evaluation of the rectum in children with extrahepatic portal venous obstruction. J Pediatr Gastroenterol Nutr. 1996;23(4):438-41.

28. Dhiman RK, Choudhuri G, Saraswat VA, Mukhopadhyay DK, Khan EM, Pandey R, et al. Endoscopic ultrasonographic evaluation of the rectum in cirrhotic portal hypertension. Gastrointest Endosc. 1993;39(5):635-40.

29. Dhiman RK, Saraswat VA, Choudhuri G, Sharma BC, Pandey R, Naik SR. Endosonographic, endoscopic, and histologic evaluation of alterations in the rectal venous system in patients with portal hypertension. Gastrointest Endosc. 1999;49(2):218-27.

30. Wiechowska-Kozłowska A, Białek A, Milkiewicz P. Prevalence of "deep" rectal varices in patients with cirrhosis: an EUS-based study. Liver Int. 2009;29(8):1202-5.

31. Sato T, Yamazaki K, Akaike J. Evaluation of the hemodynamics of rectal varices by endoscopic ultrasonography. J Gastroenterol. 2006;41(6):588-92.

32. Sato T, Yamazaki K, Toyota J, Karino Y, Ohmura T, Akaike J. Diagnosis of rectal varices via color Doppler ultrasonography. Am J Gastroenterol. 2007;102(10):2253-8.

33. Sato T, Yamazaki K, Akaike J. Evaluation of the hemodynamics of rectal varices by endoscopic ultrasonography. J Gastroenterol. 2006 Jul:41(6):588-92.

34. Katz JA, Rubin RA, Cope C, Holland G, Brass CA. Recurrent bleeding from anorectal varices: successful treatment with a transjugular intrahepatic portosystemic shunt. Am J Gastroenterol. 1993;88(7):1104-7.

35. Turon F, Casu S, Hernández-Gea V, Garcia-Pagán JC. Variceal and other portal hypertension related bleeding. Best Pract Res Clin Gastroenterol. 2013;27(5):649-64.

36. Cárdenas A, Ginès P, Uriz J, Bessa X, Salmerón JM, Mas A, et al. Renal failure after upper gastrointestinal bleeding in cirrhosis: incidence, clinical course, predictive factors, and short-term prognosis. Hepatology. 2001;34(4 Pt 1):671-6.

37. Villanueva C, Colomo A, Bosch A, Concepcion M, Hernandez-Gea $\mathrm{V}$, Aracil C, et al. Transfusion strategies for acute upper gastrointestinal bleeding. N Engl J Med. 2013;368:11-21.

38. Wells M, Chande N, Adams P, Beaton M, Levstik M, Boyce E, et al. Meta-analysis: vasoactive medications for the management of acute variceal bleeds. Aliment Pharmacol Ther. 2012;35(11):1267-78.

39. Sarin SK, Kumar A, Angus PW, Baijal SS, Baik SK, Bayraktar Y, et al. Diagnosis and management of acute variceal bleeding: Asian Pacific Association for Study of the Liver recommendations. Hepatol Int. 2011;5(2):607-24.

40. Garcia-Tsao G, Sanyal AJ, Grace ND, Carey W, Shuhart MC, Davis GL, et al. Prevention and management of gastroesophageal varices and variceal hemorrhage in cirrhosis. Hepatology. 2007;46:922-38.

41. Lee YY, Tee H-P, Mahadeva S. Role of prophylactic antibiotics in cirrhotic patients with variceal bleeding. World J Gastroenterol. 2014;20(7):1790-6.

42. Hou M-C, Lin H-C, Liu T-T, Kuo BI-T, Lee F-Y, Chang F-Y, et al. Antibiotic prophylaxis after endoscopic therapy prevents rebleeding in acute variceal hemorrhage: a randomized trial. Hepatology. 2004;39(3):746-53.

43. Bernard B, Grangé JD, Khac EN, Amiot X, Opolon P, Poynard T. Antibiotic prophylaxis for the prevention of bacterial infections in cirrhotic patients with gastrointestinal bleeding: a meta-analysis. Hepatology. 1999;29(6):1655-61.
44. Wang M, Desigan G, Dunn D. Endoscopic sclerotherapy for bleeding rectal varices: a case report. Am J Gastroenterol. 1985;80(10):779-80.

45. Weiserbs DB, Zfass AM, Messmer J. Control of massive hemorrhage from rectal varices with sclerotherapy. Gastrointest Endosc. 1986;32(6):419-21.

46. Richon J, Berclaz R, Schneider PA, Marti MC. Sclerotherapy of rectal varices. Int J Color Dis. 1988;3(2):132-4.

47. Yamanaka T, Shiraki K, Ito T, Sugimoto K, Sakai T, Ohmori S, et al. Endoscopic sclerotherapy (ethanolamine oleate injection) for acute rectal varices bleeding in a patient with liver cirrhosis. HepatoGastroenterology. 2002;49(46):941-3.

48. Sato T, Yamazaki K, Akaike J, Toyota J, Karino Y, Ohmura T. Retrospective analysis of endoscopic injection sclerotherapy for rectal varices compared with band ligation. Clin Exp Gastroenterol. 2010;3:159-63.

49. Kojima T, Onda M, Tajiri T, Kim DY, Toba M, Masumori K, et al. A case of massive bleeding from rectal varices treated with endoscopic variceal ligation (EVL). Nihon Shokakibyo Gakkai Zasshi. 1996;93(2):114-9.

50. Uno Y, Munakata A, Ishiguro A, Fukuda S, Sugai M, Munakata H. Endoscopic ligation for bleeding rectal varices in a child with primary extrahepatic portal hypertension. Endoscopy. 1998;30(9): S107-8.

51. Firoozi B, Gamagaris Z, Weinshel EH, Bini EJ. Case report: endoscopic band ligation of bleeding rectal varices. Dig Dis Sci. 2002;47(7):1502-5.

52. Sato T, Yamazaki K, Toyota J, Karino Y, Ohmura T, Suga T. Two cases of rectal varices treated by endoscopic variceal ligation. Dig Endosc. 1999;11(1):66-9.

53. Shudo R, Yazaki Y, Sakurai S, Uenishi H, Yamada H, Sugawara K. Endoscopic variceal ligation of bleeding rectal varices: a case report. Dig Endosc. 2000;12(4):366-8.

54. Coelho-Prabhu N, Baron T, Kamath P. Endoscopic band ligation of rectal varices: a case series. Endoscopy. 2010;42(2):173-6.

55. Chen WC, Hou MC, Lin HC, Chang FY, Lee SD. An endoscopic injection with N-butyl-2-cyanoacrylate used for colonic variceal bleeding: a case report and review of the literature. Am J Gastroenterol. 2000;95(2):540-2.

56. Weilert F, Shah JN, Marson FP, Binmoeller KF. EUS-guided coil and glue for bleeding rectal varix. Gastrointest Endosc. 2012;76(4): 915-6.

57. Sharma M, Somasundaram A. Massive lower GI bleed from an endoscopically inevident rectal varices: diagnosis and management by EUS (with videos). Gastrointest Endosc. 2010;72(5):1106-8.

58. Sarin SK, Kumar A. Endoscopic treatment of gastric varices. Clin Liver Dis. 2014;18(4):809-27.

59. Tripathi D, Ferguson JW, Therapondos G, Plevris JN, Hayes PC. Review article: recent advances in the management of bleeding gastric varices. Aliment Pharmacol Ther. 2006;24(1):1-17.

60. Messallam A, Kumbhari V, Saxena P, Azola A, Kalloo A, Khashab M. Large bleeding rectal varices treated with endoscopic ultrasound-guided coiling and cyanoacrylate injection. Endoscopy. 2014;46(S 01):E28-9.

61. Philips CA, Augustine P. Endoscopic ultrasound-guided management of bleeding rectal varices. ACG Case Rep J. 2017;4:e101.

62. Connor EK, Duran-Castro OL, Attam R. Therapy for recurrent bleeding from rectal varices by EUS-guided sclerosis. Gastrointest Endosc. 2015;81(5):1280-1.

63. Seewald S, Ang TL, Imazu H, Naga M, Omar S, Groth S, et al. A standardized injection technique and regimen ensures success and safety of N-butyl-2-cyanoacrylate injection for the treatment of gastric fundal varices (with videos). Gastrointest Endosc. 2008;68(3): 447-54.

64. McAvoy NC, Plevris JN, Hayes PC. Human thrombin for the treatment of gastric and ectopic varices. World J Gastroenterol. 2012;18(41):5912-7. 
65. Ibukuro K, Kojima K, Kigawa I, Tanaka R, Fukuda H, Abe S, et al. Embolization of rectal varices via a paraumbilical vein with an abdominal wall approach in a patient with massive ascites. J Vasc Interv Radiol. 2009;20(9):1259-61.

66. Ahn SS, Kim EH, Kim MD, Lee WJ, Kim SU. Successful hemostasis of intractable rectal variceal bleeding using variceal embolization. World J Gastroenterol. 2015;21(8):2558-62.

67. Fantin AC, Zala G, Risti B, Debatin JF, Schöpke W, Meyenberger C. Bleeding anorectal varices: successful treatment with transjugular intrahepatic portosystemic shunting (TIPS). Gut. 1996;38(6):932-5.

68. Ory G, Spahr L, Megevand JM, Becker C, Hadengue A. The longterm efficacy of the intrahepatic portosystemic shunt (TIPS) for the treatment of bleeding anorectal varices in cirrhosis. A case report and review of the literature. Digestion. 2001;64(4):261-4.

69. Godil A, McCracken JD. Rectal variceal bleeding treated by transjugular intrahepatic portosystemic shunt. Potentials and pitfalls. J Clin Gastroenterol. 1997;25(2):460-2.

70. Haskal ZJ, Scott M, Rubin RA, Cope C. Intestinal varices: treatment with the transjugular intrahepatic portosystemic shunt. Radiology. 1994;191(1):183-7.

71. Vangeli M, Patch D, Terreni N, Tibballs J, Watkinson A, Davies N, et al. Bleeding ectopic varices - treatment with transjugular intrahepatic porto-systemic shunt (TIPS) and embolisation. J Hepatol. 2004;41(4):560-6.

72. Tripathi D, Jalan R. Transjugular intrahepatic portosystemic stentshunt in the management of gastric and ectopic varices. Eur J Gastroenterol Hepatol. 2006;18(11):1155-60.

73. Tripathi D, Helmy A, Macbeth K, Balata S, Lui HF, Stanley AJ, et al. Ten years' follow-up of 472 patients following transjugular intrahepatic portosystemic stent-shunt insertion at a single centre. Eur J Gastroenterol Hepatol. 2004;16(1):9-18.

74. Vidal V, Joly L, Perreault P, Bouchard L, Lafortune M, PomierLayrargues G. Usefulness of transjugular intrahepatic portosystemic shunt in the management of bleeding ectopic varices in cirrhotic patients. Cardiovasc Intervent Radiol. 2006;29(2):216-9.

75. Kochar N, Tripathi D, McAvoy NC, Ireland H, Redhead DN, Hayes PC. Bleeding ectopic varices in cirrhosis: the role of transjugular intrahepatic portosystemic stent shunts. Aliment Pharmacol Ther. 2008;28(3):294-303.

76. Kanagawa H, Mima S, Kouyama H, Gotoh K, Uchida T, Okuda K. Treatment of gastric fundal varices by balloon-occluded retrograde transvenous obliteration. J Gastroenterol Hepatol. 1996;11(1):51-8.
77. Al-Osaimi AMS, Caldwell SH. Medical and endoscopic management of gastric varices. Semin Intervent Radiol. 2011;28(3):273-82.

78. Triantafyllou M, Stanley AJ. Update on gastric varices. World J Gastrointest Endosc. 2014;6(5):168-75.

79. Choi YH, Yoon CJ, Park JH, Chung JW, Kwon JW, Choi GM. Balloon-occluded retrograde transvenous obliteration for gastric variceal bleeding: its feasibility compared with transjugular intrahepatic portosystemic shunt. Korean J Radiol. 2003;4(2): 109-16.

80. Saad WEA. Balloon-occluded retrograde transvenous obliteration of gastric varices: concept, basic techniques, and outcomes. Semin Intervent Radiol. 2012;29(2):118-28.

81. Matsumoto A, Hamamoto N, Nomura T, Hongou Y, Arisaka Y, Morikawa $\mathrm{H}$, et al. Balloon-occluded retrograde transvenous obliteration of high risk gastric fundal varices. Am J Gastroenterol. 1999;94(3):643-9.

82. Akahane T, Iwasaki T, Kobayashi N, Tanabe N, Takahashi N, Gama $\mathrm{H}$, et al. Changes in liver function parameters after occlusion of gastrorenal shunts with balloon-occluded retrograde transvenous obliteration. Am J Gastroenterol. 1997;92(6):1026-30.

83. Fukuda T, Hirota S, Sugimura K. Long-term results of balloonoccluded retrograde transvenous obliteration for the treatment of gastric varices and hepatic encephalopathy. J Vasc Interv Radiol. 2001;12(3):327-36.

84. Akhter NM, Haskal ZJ. Diagnosis and management of ectopic varices. Gastrointest Interv. 2012;1(1):3-10.

85. Yoshino K, Imai Y, Nakazawa M, Chikayama T, Ando S, Sugawara $\mathrm{K}$, et al. Therapeutic strategy for patients with bleeding rectal varices complicating liver cirrhosis. Hepatol Res. 2014;44(11):1088-94.

86. Biswas S, George ML, Leather AJM. Stapled anopexy in the treatment of anal varices: report of a case. Dis Colon Rectum. 2003;46(9):1284-5.

87. Botterill ID, Jayne DG, Snelling AP, Ambrose NS. Correction of symptomatic ano-rectal varices with circumferential stapled anoplasty. Color Dis. 2002;4(3):217.

88. Kaul AK, Skaife PG. Circumferential stapled procedure for bleeding ano-rectal varices is an effective treatment-experience in nine patients. Color Dis. 2009;11(4):420-3.

89. Yeh $\mathrm{T}, \mathrm{McGuire} \mathrm{HH}$. Intractable bleeding from anorectal varices relieved by inferior mesenteric vein ligation. Gastroenterology. 1994;107(4):1165-7.

90. Sarin SK, Kumar CKN. Ectopic varices. Clin Liver Dis. 2012;1(5): 168-72. 